\title{
Prevalence of inter-hemispheric asymetry in children and adolescents with interdisciplinary diagnosis of non-verbal learning disorder
}

\author{
Prevalência de assimetria inter-hemisférica em crianças e adolescentes com \\ diagnóstico interdisciplinar de transtorno da aprendizagem não verbal
}

\author{
Alessandra Bernardes Caturani Wajnsztejn ${ }^{1}$, Bianca Bianco ${ }^{1}$, Caio Parente Barbosa ${ }^{1}$
}

\begin{abstract}
Objective: To describe clinical and epidemiological features of children and adolescents with interdisciplinary diagnosis of nonverbal learning disorder and to investigate the prevalence of interhemispheric asymmetry in this population group. Methods: Crosssectional study including children and adolescents referred for interdisciplinary assessment with learning difficulty complaints, who were given an interdisciplinary diagnosis of non-verbal learning disorder. The following variables were included in the analysis: sex-related prevalence, educational system, initial presumptive diagnoses and respective prevalence, overall non-verbal learning disorder prevalence, prevalence according to school year, age range at the time of assessment, major family complaints, presence of inter-hemispheric asymmetry, arithmetic deficits, visuoconstruction impairments and major signs and symptoms of non-verbal learning disorder. Results: Out of 810 medical records analyzed, 14 were from individuals who met the diagnostic criteria for non-verbal learning disorder, including the presence of inter-hemispheric asymmetry. Of these 14 patients, 8 were male. Conclusion: The high prevalence of inter-hemispheric asymmetry suggests this parameter can be used to predict or support the diagnosis of non-verbal learning disorder.
\end{abstract}

Keywords: Learning disorders/diagnosis; Child; Adolescent

\section{RESUMO}

Objetivo: Descrever as características clínicas e epidemiológicas de crianças e adolescentes com transtorno de aprendizagem não verbal, e investigar a prevalência de assimetria inter-hemisférica neste grupo populacional. Métodos: Estudo transversal que incluiu crianças e adolescentes encaminhados para uma avaliação interdisciplinar, com queixas de dificuldades de aprendizagem e que receberam diagnóstico interdisciplinar de transtorno de aprendizagem não verbal. As variáveis avaliadas foram prevalência por sexo, sistema de ensino, hipóteses diagnósticas iniciais e respectivas prevalências, prevalência de condições em relação à amostra total, prevalência geral do transtorno de aprendizagem não verbal, prevalência de acordo com ano escolar, faixa etária no momento da avaliação, principais queixas familiares, presença assimetria inter-hemisférica, dificuldade em aritmética, alterações em visuoconstrução, e principais sinais e sintomas do transtorno de aprendizagem não verbal. Resultados: Dos 810 prontuários médicos analisados, 14 eram de indivíduos que preencheram os critérios diagnósticos para transtorno de aprendizagem não verbal, incluindo a assimetria inter-hemisférica. Destes 14 pacientes, 8 eram do sexo masculino. Conclusão: A alta prevalência de assimetria inter-hemisférica sugere que este parâmetro possa ser usado como preditor ou reforçador para diagnóstico de transtorno de aprendizagem não verbal.

Descritores: Transtornos de aprendizagem/diagnóstico; Criança; Adolescente

\section{INTRODUCTION}

The study of learning difficulties dates back from the $19^{\text {th }}$ century and was motivated by the recognition of reading difficulties unrelated to intellectual ability ${ }^{(1)}$ and sensory deficits, level of instruction or learning motivation. The umbrella term "learning disability"(2,3) comprises a group of specific and non-specific disorders affecting reading, writing, math and other basic learning skills. Such disorders are characterized by distinct signs and symptoms manifested by children with learning

\footnotetext{
Faculdade de Medicina do ABC, Santo André, SP, Brazil.

Corresponding author: Bianca Bianco - Avenida Príncipe de Gales, 821 - Vila Príncipe de Gales - Zip code: 09060-650 - Santo André, SP, Brazil - Phone: (55 11) $4993-5464$ E-mail: bianca.bianco@hotmail.com

Received on: Apr 20, 2016 - Accepted on: Aug 19, 2016

Conflict of interest: none.
}

DOI: 10.1590/\$1679-45082016A03722 
difficulties, which keep them from achieving their full potential. $^{(4)}$

The generic term "learning disabilities", proposed by the National Joint Committee on Learning Disabilities, ${ }^{(5)}$ in 1988, refers to a heterogeneous group of disorders characterized by significant difficulties in the acquisition and application of speaking, reading, writing, reasoning and math skills. These lifelong disorders, which are intrinsic to the affected individual, are presumably due to central nervous system dysfunctions. Affected individuals may have concurrent behavior regulation (otherwise known as self-regulation), perception and social competence problems. Another form of learning disability, ${ }^{(6,7)}$ referred to as non-verbal disability, has been proposed to describe children who do not have severe language problems and are able to acquire reading and writing skills, but present with "persistent deficits in right-left orientation and difficulties with constructional tasks and arithmetic, whose deficits are non-verbal and who are unable to grasp the significance of many aspects of the environment".

There are no population-based studies addressing the prevalence of non-verbal learning disorder (NVLD). Still, learning disabilities are thought to affect an estimated $10 \%$ of school-age children, with $1 \%$ prevalence of NVLD in this group, ${ }^{(8)}$ and to account for an estimated $10 \%$ of learning-disability-related medical appointments. This study revealed a significant NVLD prevalence. Data presented are expected to inform healthcare and education professionals about the disorder, to prevent false-negative diagnoses.

The major feature of NVLD is the discrepancy between verbal and non-verbal (or performance) intelligence quotients (IQ), controlled by the left and right brain hemispheres, respectively.(9,10) "Attention deficits and other more general forms of neurological impairments may actually by masked by information processing deficits that occur with advancing age". ${ }^{(1)}$

Different sets of criteria can be employed to evaluate individuals with $\mathrm{NVLD},{ }^{(12,13)}$ such as the Wechsler Intelligence Scale for Children with verbal IQ greater than 79, and tests aimed to identify deficits in tactile and visual perception, complex psychomotor activity and the ability to deal with new materials, which gave rise to the profile matching algorithm developed by the authors.

Non-verbal learning disorder signs and symptoms do not match those of any well-described condition; therefore, difficulties in characterizing this condition remain to this day. ${ }^{(14)}$ Non-verbal learning disorder is a specific condition characterized by difficulties in motor coordination, somatosensory perception, visuospatial cognition, inductive and arithmetic reasoning, cognition and social skills. ${ }^{(12,15)}$

The three major areas of dysfunction in NVLD include motor and visuospatial abilities, organizational skills and social skills. ${ }^{(16)}$

Children and adolescents with NVLD often have good reading and writing skills, but have difficulties with inferential reasoning, reading comprehension and math. ${ }^{(17)}$

Affected children have difficulties understanding cause-effect relationships, poor reasoning and problem solving skills and difficulties understanding complex or abstract ideas, and therefore tend to have executive functioning issues. ${ }^{(17,18)}$

The diagnosis of NVLD is based on the identification of deficits in social perception, social judgment and social interaction skills, ${ }^{(19)}$ given such difficulties are secondary to impaired visuospatial development ${ }^{(20,21)}$ and pervade diagnostic strategies based on recognition of significant perception problems, faulty understanding of facial expressions, tone of voice and speaker's intention. ${ }^{(22-25)}$

Low scores in specific motor performance tests involving both hands suggest bilateral brain involvement and confirm the presence of motor coordination impairments, with worse performance in the right hemisphere compared to the left. Children with NVLD are described as clumsy and uncoordinated. ${ }^{(25,26)}$

Visuospatial deficits are the major characteristic of children with NVLD, even in the absence of severe motor problems. Differences between verbal and performance (non-verbal) IQ scores are not a requisite for the diagnosis of NLVD; still this finding has been particularly emphasized in affected children. ${ }^{(26)}$

The neuropsychological model of NVLD ${ }^{(27)}$ accounts for strengths and weaknesses in the child's skills profile, and describes some deficits or skills as essential, while others are thought to be of secondary importance.

Hence, difficulties in the identification and characterization of individuals affected with NVLD remain, as do difficulties in confirmation and quantification of motor, socialization and learning deficits, and interhemispheric asymmetry. According to a literature review, ${ }^{(28)}$ interhemispheric asymmetry, deficits in fine motor and visuoconstructive coordination, difficulties with visuospatial memory tasks and math, and social emotional impairments are the most significant factors in NVLD. A magnetic resonance imaging study ${ }^{(29)}$ comparing different portions of the corpus callosum revealed significantly smaller splenium in children affected with NVLD compared to children in all other groups studied (control children, children affected with attention deficit hyperactivity disorder predominantly 
inattentive and others, and children with highfunctioning autism). Findings in the NVLD group in that study were associated with low performance IQ, but not with low verbal IQ scores.

\section{OBJECTIVE}

To describe clinical and epidemiological features of children and adolescents with interdisciplinary diagnosis of non-verbal learning disorder and to investigate the prevalence of inter-hemispheric asymmetry in this population group.

\section{METHODS}

MEDLINE and ScienceDirect databases were searched using PubMed and Scopus search engines respectively. The keywords "child", "adolescent" and "non-verbal learning disorder" were used to locate related articles and respective references in PubMed (US National Library of Medicine/National Institutes of Health). Only publications with title or abstract in Portuguese or English were included in the analysis.

This is a cross-sectional study based on data extracted from medical records of patients seen at the Núcleo Especializado em Aprendizagem da Faculdade de Medicina do ABC from 2008 to 2014. All patients were children or adolescents submitted to interdisciplinary assessment with learning difficulty complaints and diagnosed with NVLD. The following variables were included in the analysis: sex-related prevalence, educational system (i.e., public or private school attendance), initial presumptive diagnosis and respective prevalence, overall NVLD prevalence, NVLD prevalence according to school year, age range at the time of assessment, major family complaints, presence of interhemispheric asymmetry, arithmetic deficits and visuoconstruction impairments, and major NVLD signs and symptoms.

The diagnosis was made by a multidisciplinary team via an interdisciplinary assessment protocol applied to students attending private and public elementary schools in the $\mathrm{ABC}$ region, State of São Paulo, Brazil. Patients were individually evaluated; cases, medical opinion on the test protocol and interdisciplinary NVLD diagnostic decision making were then discussed in meetings held at the end of the data collection period. This study was approved by the Research Ethics Committee, protocol 471.336, CAAE: 17200213.3.0000.0082.

The interdisciplinary evaluation comprised 14 appointments, as follows: medical history, screening at the Pediatric Neurology unit, three neuropsychology sessions, two speech therapy sessions, two psychology/ psychopedagogy sessions, one interdisciplinary clinical discussion session, one report preparation session and a final feedback session with the patient's legal guardian. The interdisciplinary protocol was systematically applied to all of 810 subjects in the sample. The following instruments were used:(30-33) Attention deficit hyperactivity disorder, Children's Apperception Test, Phonological awareness: instrument of sequential assessment (Consciência fonológica: instrumento de avaliação sequencial), Verbal Fluency Test (FAS), House-Tree-Person test, reading comprehension, Rey Complex Figure Test and Recognition Trial, MTASNAP-IV scale for evaluation of symptoms of attentiondeficit/ hyperactivity disorder and oppositionaldefiant disorder, Perceived Stress Scale, Thematic Apperception Test, Academic Achievement Test, TIPITI Language Assessment and the third edition of the Weschler Intelligence Scale for Children III.

Non-verbal learning disorder diagnosis was based on the following criteria: bilateral deficits in tactile perception, usually more pronounced on the left side of the body; bilateral deficits in psychomotor coordination; compromised visuospatial organizational skills; significant difficulties in dealing with new or complex situations and pieces of information; significant deficits in nonverbal problem solving, concept building and hypothesis testing; perceptual and temporal orientation distortion; well-developed verbal memorization skills; routine and repetitive verbosity, language content distortion and pragmatic communication impairments; significant deficits in arithmetic and reading comprehension with intact isolated word reading ability; significant perceptual, judgment and social interaction deficits, often leading to social isolation.

Inclusion criteria were as follows: children and adolescents diagnosed with NVLD regardless of drug treatment, referred for interdisciplinary assessment with learning difficulty complaints. Exclusion criteria comprised central nervous system malformations, neurological syndromes and intellectual disabilities.

Descriptive statistical analysis was based on absolute and relative frequencies and measures of central tendency and dispersion. Statistical analyses were performed using software Epi Info.

\section{RESULTS}

Data on sex-related prevalence, educational system, suspicion of NVLD, NVLD prevalence following interdisciplinary assessment and school grade of the 14 
patients diagnosed with NVLD in this sample are given in table 1; initial presumptive diagnosis and respective prevalence are given in table 2.

Family complaints associated with the initial presumptive diagnosis were major factors in comorbidity identification, as shown in table 3 .

Table 1. Sex-related prevalence, educational system, school year, referral initiative and initial non-verbal learning disorder suspicion

\begin{tabular}{|c|c|c|}
\hline & $\begin{array}{c}\text { Higher incidence } \\
\text { n (\%) }\end{array}$ & $\begin{array}{l}\text { Lower incidence } \\
\text { n (\%) }\end{array}$ \\
\hline Sex-related prevalence & 589 boys $(72.71)$ & 221 girls (27.28) \\
\hline Educational system & 679 private $(83.82)$ & 131 public (16.17) \\
\hline Initial NVLD suspicion & 808 not suspected (99.76) & 2 suspected $(0.24)$ \\
\hline NVLD prevalence & Non-NVLD 806 (98.28) & 14 cases $(1.72)$ \\
\hline \multirow[t]{2}{*}{ School stage } & 12 cases in & 2 cases in \\
\hline & High School (85.71) & Primary School (14.29) \\
\hline $\begin{array}{l}\text { Gender prevalence in the } \\
\text { NLVD sample }\end{array}$ & 8 boys $(57.15)$ & 6 girls (42.85) \\
\hline $\begin{array}{l}\text { Referral for } \\
\text { interdisciplinary } \\
\text { assessment }\end{array}$ & $\begin{array}{c}11 \text { cases referred by health } \\
\text { professionals }(78.57)\end{array}$ & $\begin{array}{c}3 \text { cases referred by other } \\
\text { professionals }(21.43)\end{array}$ \\
\hline
\end{tabular}

Table 2. Initial presumptive diagnoses made by referring professionals, with complaints of learning disabilities and respective prevalence

\begin{tabular}{lc}
\hline Autism spectrum disorder & 1 \\
Intellectual disability & 1 \\
Non-verbal learning disorder & 2 \\
Attention deficit/ hyperactivity disorder predominantly inattentive & 4 \\
Learning disability & 6 \\
Number of cases diagnosed with non-verbal learning disorder & 14 \\
\hline
\end{tabular}

Table 3. Family complaints associated reported

\begin{tabular}{lc}
\hline Major family complaints & Incidence \\
\hline Autism spectrum disorder & 1 \\
Immaturity & 1 \\
Anxiety & 1 \\
Inattention & 5 \\
Motor & 9 \\
Socialization & 11 \\
Ingenuity & 13 \\
Shyness & 14 \\
Communication & 14 \\
Learning & 14 \\
\hline
\end{tabular}

Interhemispheric asymmetry was a common finding in NVLD patients (Table 4).

As an aside, arithmetic and Rey Complex Figure Test and Recognition Trial test results are shown in table 5.

Table 4. Interhemispheric asymmetry

\begin{tabular}{lc}
\hline Non-verbal learning disorder & $\mathbf{1 4}$ \\
\hline Intelligence quotient $>69$ & 14 \\
Borderline total intelligence quotient $\leq 79$ & 2 \\
Borderline verbal intelligence quotient $>79$ & 14 \\
Performance intelligence quotient dysfunction & 2 \\
Borderline performance intelligence quotient & 7 \\
Performance intelligence quotient below average & 3 \\
Average performance intelligence quotient & 2 \\
Interhemispheric asymmetry & 14 \\
\hline
\end{tabular}

Table 5. Arithmetic and Rey Complex Figure tests

\begin{tabular}{lccc}
\hline Arithmetic & Incidence & Rey figure & Incidence \\
\hline Average & 4 & Average & 1 \\
Below average & 5 & Below average & 8 \\
Borderline & 5 & Borderline & 3 \\
Dysfunction & 0 & Dysfunction & 2 \\
\hline
\end{tabular}

\section{DISCUSSION}

Non-verbal learning disorder research has seen significant advances since $1964{ }^{(6)}$ Following a similar path, this study addressed the definition of NVLD-specific criteria for clinical characterization of NVLD.

Non-verbal learning disorder has been described ${ }^{(5)}$ as a learning disability subtype and is thought to be a form of pervasive developmental disorder. ${ }^{(34)}$ These possibilities have not been listed in the fifth edition of Diagnostic and Statistical Manual of Mental Disorders (DSM-5), published in 2013, ${ }^{(35)}$ and remain to be confirmed.

Visuospatial impairments, tactile and motor deficits are the major criteria for learning disorder diagnosis. ${ }^{(6,20,21)}$

One of the most important NVLD diagnostic criteria is the presence of interhemispheric asymmetry in association with low performance intelligence quotient scores and attention deficits, which tend to decrease with neurological development. ${ }^{(9,10,29)}$

Other criteria, such as right-left orientation issues, construction and nonverbal arithmetic difficulties, and 
difficulties to grasp the meaning of many aspects of the environment have also been described. ${ }^{(5)}$

The following criteria were proposed by Hale, ${ }^{(14)}$ deficits in motor coordination, perceptual and somatosensory deficits, visuospatial impairments, difficulties with inductive and arithmetic reasoning, and impaired cognitive and social skills.

Secondary and tertiary deficits associated with NVLD were described and would include delayed acquisition or failure to acquire the necessary ability to perform tasks, such as riding a bicycle or tying shoe laces, graphic renditions with infantile characteristics (e.g., inconsistent strokes), impaired spatial organization, difficulties memorizing multiplication tables and executing complex arithmetic operations (such as algorithms), shyness, social awkwardness, ingenuity, little or no malice, faulty recognition of implicit aspects in social interactions (i.e., sarcasm, irony and metaphors), incoordination and clumsiness.

Data analysis revealed similar gender-related prevalence in the sample studied ( 8 boys versus 6 girls affected with NVLD). Similar data have been reported elsewhere. ${ }^{(25)}$

The relationship between NVLD and educational system has not been described in literature. This study revealed higher NVLD incidence in private schools; however, this finding might be explained by the fact that children in this sample attended predominantly private schools.

The prevalence of NVLD in the overall child/ adolescent population has not been reported; still, $1 \%$ of patients with learning disorders are thought to suffer from NVLD. A $1.72 \%$-prevalence of NVLD was documented in this study.

As regards the initial evaluation by referring professionals, this study revealed that $64.28 \%$ of patients were referred by healthcare professionals, particularly physicians.

In this study, only $14.28 \%$ of 14 patients diagnosed with NVLD were suspected of the disorder. Out of 810 cases included in the sample, 12 were false negative, and there were no false positive results.

Data analysis revealed that $85.52 \%$ of patients diagnosed NVLD were in high school at the time of interdisciplinary assessment. Similar findings have been reported in literature. ${ }^{(14)}$ Data of this study also suggest that affected patients tend seek advice around the beginning of the middle school cycle; increased interpretation, logical thinking and socialization demands in this phase tend to exacerbate NVLD signs and symptoms.

Age group analysis revealed a trend towards diagnosis at 12 to 13 years of age, again coinciding with the beginning of the high school cycle.
Three significant aspects related to family complaints reported during anamnesis were emphasized in this study. Shyness, learning and communication deficits were reported in all 14 NVLD cases. Naive thoughts and socialization difficulties were reported by 13 and 11 families, respectively. Nine families reported fine, global or gross motor deficits.

Awkwardness, faulty understanding of implicit aspects and difficulties associated with inferential reasoning, interpretation, production of spontaneous or direct speech, receptivity and prosody of speech were present in all 14 NVLD cases submitted to interdisciplinary assessment in this study.

As in previous studies, ${ }^{(9)}$ interhemispheric asymmetry with low performance intelligence quotient scores and total intelligence quotient scores above 69 was a consistent finding in NVLD patients in this sample.

Difficulties with arithmetic, logical reasoning and visuospatial organization have been reported in earlier NVLD studies. ${ }^{(9)}$ Patients in this sample scored average, below average or borderline (4, 5 and 5 out of 14 cases, respectively) in the arithmetic subtest of Weschler Intelligence Scale for Children III. Rey Complex Figure Test and Recognition Trial scores of 14 patients were as follows: one average, eight below average, three borderline and two dysfunction. Results of both tests were consistent with findings reported by other researchers. ${ }^{(26)}$

As regards the psychosocial impact on NVLD, given their apparently normal linguistic performance, affected children and adolescents are often expected to perform beyond their actual abilities. Such unrealistic expectations inevitably lead to frustration. The psychosocial impacts of NVLD may be exacerbated by comorbidities, such as externalizing (preschool age children) and internalizing disorders (from preadolescence).

\section{CONCLUSION}

This study revealed a significant prevalence of non-verbal learning disorder and highlighted relevant aspects of the disease. Data presented provide significant contribution to the definition of non-verbal learning disorder diagnostic criteria. Confirmation of interhemispheric asymmetry in all 14 patients diagnosed with non-verbal learning disorder in this sample suggests this parameter can be used to predict or support the diagnosis of the condition.

Growing evidences of non-verbal learning disorder suggest the condition deserves closer attention on the part of health and education professionals, as the knowledge acquisition process is an arduous journey for individuals with learning and communication. 
Non-verbal learning disorder signs and symptoms overlap with those of other conditions, such as social pragmatic communication and autism spectrum disorders. Therefore, disclosure of reliable data on non-verbal learning disorder will certainly contribute to faulty diagnosis mitigation and provision of better management guidelines, with positive impacts on the quality of life of affected patients and their families.

The study of the trajectory of patients affected with non-verbal learning disorder and the characterization of disease-related impacts comprise a highly relevant topic for future research aimed at preventing the development of comorbidities, such as anxiety and depression.

Investigation of the impact of bullying against individuals with non-verbal learning disorder is vital, given this form of suffering is a major triggering factor for anxiety, depression, phobia, social isolation and poor academic performance.

Further research into the relations between teaching and learning and the development of alternative evaluation methods adapted to individuals affected with non-verbal learning disorder is warranted, given they tend to learn more through repetition than intuitive or inductive experience.

Family disease history investigation may aid in the identification of potential underlying disease mechanisms, as may the investigation of maternal prenatal, perinatal and postnatal care. The recognition of biomarkers, genetic and hereditary factors may also promote the development of case-specific interventions and more accurate prognostication. Research data can be applied to the development of prevention strategies and actions aimed to decrease the socioeconomic impacts of non-verbal learning disorder on the public and private healthcare systems.

\section{CONTRIBUTIONS}

Alessandra Bernardes Caturani Wajnsztejn conceived and designed the study and was in charge of data collection, analysis and interpretation.

Bianca Bianco critically reviewed this manuscript and granted final approval for submission.

Caio Parente Barbosa conceived and designed the study, critically reviewed this manuscript and granted final approval for submission.

\section{REFERENCES}

1. Goddard HH. Four hundred feeble-minded children classified by the Binet method. 1910. J Genet Psychol. 1991;152(4):437-47.

2. Orton ST. Reading, writing and speech problems in children. New York: Norton; 1937.
3. Kirk SA. Research on the education of the mentally retarded. Slow Learn Child 1955;1(3):96-102.

4. Rie ED, Rie HE, Stewart S, Rettemnier SC. An analysis of neurological soft signs in children with learning problems. Brain Lang. 1978:6(1):32-46.

5. Hammill DD, Leigh JE, McNutt G, Larsen SC. A new definition of learning disabilities. Learn Disabil 0. 1988;11(3):217-23.

6. Boshes B, Myklebust HR. A neurological and behavioral study of children with learning disorders. Neurology. 1964;14:7-12.

7. Coles G. The learning-disabilities Test Battery: empirical and social issues. Harv Educ Rev. 1978;48(3):313-40.

8. Rourke BP. Syndrome of nonverbal learning disabilities: the final common pathway of white-matter disease/dysfunction? Clin Neuropsychol. 1987;1 (3): 209-34.

9. Rourke BP, Telegdy GA. Lateralizing significance of WISC verbal-performance discrepancies for older children with learning disabilities. Percept Mot Skills. 1971;33(3):875-83.

10. Rourke BP, Dietrich DM, Young GC. Significance of WISC verbal-performance discrepancies for younger children with learning disabilities. Percept Mot Skills. 1973;36(1):275-82

11. Cassar AG, Jang EE. Investigating the effects of a game-based approach in teaching word recognition and spelling to students with reading disabilities and attention deficits. Aust J Learn Diffic. 2010;15(2):193-211.

12. Myklebust HR. Nonverbal learning disabilities: assessment and intervention. In: Myklebust HR, editor. Progress in learning disabilities. Vol. 3. New York: Grune \& Stratton; 1975. p.108-19.

13. Pelletier PM, Ahmad SA, Rourke BP. Classification rules for basic phonological processing disabilities and nonverbal learning disabilities: formulation and external validity. Child Neuropsychol. 2001;7(2):84-98.

14. Hale J, Alfonso V, Berninger V, Bracken B, Christo C, Clark E, et al. Critical issues in response-tointervention, comprehensive evaluation, and specific learning disabilities identification and intervention: an expert white paper consensus. Learn Disabil 0. 2010;33(3):223-36.

15. Rourke BP, Finlayson AJ. Neuropsychological significance of variations in patterns of academic performance: verbal and visuo-spatial abilities. J Abnorm Child Psychol. 1978;6(1):121-33.

16. Harnadek MC, Rourke BP. Principal identifying features of the syndrome of nonverbal learning disabilities in children. J Learn Disabil. 1994;27(3):144-54.

17. Semrud-Clikeman M. Social competence in children. J Educ Res Online. 2012; $4(1): 158-60$.

18. Semrud-Clikeman M, Hynd GW. Right hemispheric dysfunction in nonverbal learning disabilities: social, academic, and adaptive functioning in adults and children. Psychol Bull. 1990;107(2):196-209. Review.

19. Semrud-Clikeman M, Glass K. Comprehension of humor in children with nonverbal learning disabilities, reading disabilities, and without learning disabilities. Ann Dyslexia. 2008;58(2):163-80.

20. Rourke BP. Neuropsychology as a psychosocial science: implications for research and clinical practice. Can Psychol. 2008;49(1):35-41.

21. Forrest BJ. The utility of math difficulties, internalized psychopathology, and visual-spatial deficits to identify children with the nonverbal learning disability syndrome: evidence for a visualspatial disability. Child Neuropsychol. 2004;10(2):129-46.

22. Semrud-Clikeman $\mathrm{M}$, Fine JG, Bledsoe J. Comparison among children with children with autism spectrum disorder, nonverbal learning disorder and typically developing children on measures of executive functioning. J Autism Dev Disord. 2014;44(2):331-42.

23. Semrud-Clikeman M, Walkowiak J, Wilkinson A, Christopher G. Neuropsychological differences among children with Asperger syndrome, nonverbal learning disabilities, attention deficit disorder, and controls. Dev Neuropsychol. 2010;35(5):582-600.

24. Pennington BF, Ozonoff S. Executive functions and developmental psychopathology J Child Psychol Psychiatry. 1996;37(1):51-87. Review. 
25. Stuebing KK, Fletcher JM, LeDoux JM, Lyon GR, Shaywitz SE, Shaywitz BA. Validity of IQ-discrepancy classifications of reading disabilities: a meta-analysis. Am Educ Res J. 2002;39(2):469-518.

26. Rourke BP. The syndrome of nonverbal learning disabilities: developmental manifestations in neurological disease, disorder, and dysfunction. Clin Neuropsychol. 1988;2(4):293-330.

27. Mammarella IC, Cornoldi $\mathrm{C}$. An analysis of the criteria used to diagnose children with Nonverbal Learning Disability (NLD). Child Neuropsychol. 2014; 20(3):255-80. Review.

28. Barkley RA, Fischer M, Smallish L, Fletcher K. The persistence of attentiondeficit/hyperactivity disorder into young adulthood as a function of reporting source and definition of disorder. J Abnorm Psychol. 2002;111(2):279-89.

29. Fine JG, Musielak KA, Semrud-Clikeman M. Smaller splenium in children with nonverbal learning disability compared to controls, high-functioning autism and ADHD. Child Neuropsychol. 2014;20(6):641-61.
30. Noronha AP, Freitas FA. [Psychological tests, use and knowledge]. Psico. 2005;36(1):21-8. Portuguese.

31. Costa DI, Azambuja LS, Portuguez MW, Costa JC. [Neurophycological assessment in children]. J Pediatr (Rio J). 2004;80(2 Supl):S111-6. Review. Portuguese.

32. Torres DI, Ciasca SM. [Correlation between teacher's complaint and phychological evaluation in children of first grade with learning difficulties]. Rev Psicopedag. 2007;24(73):18-29. Portuguese

33. Pinheiro L, Correa J, Mousinho R. [The effectiveness of speech therapist intervention in the evaluation of learning disabilities]. Rev Psicopedag. 2012 29(89):215-25. Portuguese.

34. American Psychiatric Association. Diagnostic and Statistical Manual of Menta Disorders. 4th ed. Washington (DC): American Psychiatric Association; 2000.

35. American Psychiatric Association. Diagnostic and Statistical Manual of Menta Disorders. 5th ed. Washington (DC): American Psychiatric Association; 2013 